\title{
When Advance Directives Collide
}

\author{
Jordan Potter, PhD® and Susannah W. Lee, JD, MPH
}

Ethics Program, Wellstar Health System, Atlanta, GA, USA.

$\mathrm{J}$ Gen Intern Med 35(7):2191-2

DOI: $10.1007 / \mathrm{s} 11606-020-05680-\mathrm{x}$

(c) Society of General Internal Medicine 2020

I ncreasingly, patients are encouraged to document their fundamental values and preferences for their healthcare in legal forms known as advance directives. These documents assist patients in ensuring that their values and healthcare preferences are known and respected when they are unable to make medical decisions for themselves, especially at the end of life. While appropriate for virtually every individual at any stage of adult life, these documents are especially beneficial for those with chronic illnesses and those at the end of life, as these individuals have more frequent and substantial hospitalizations and are more likely to encounter scenarios where they are unable to make medical decisions for themselves. In recent years, advance directives have become more prevalent and utilized by patients. The most recent study in 2017 estimates that approximately $36.7 \%$ of Americans have filled out some form of advance directive, including around $38.2 \%$ of Americans with chronic illnesses. ${ }^{1}$

The two main types of advance directives are the Healthcare Power of Attorney (HcPOA) and the Living Will (LW). The HcPOA enables patients to designate a specific person to serve as their surrogate decision-maker to make medical decisions for them when they are incapable of doing so themselves, such as when unconscious or in a minimally conscious state. The LW allows patients to document specific treatment preferences that they have regarding types of healthcare that they do and do not want, particularly life-sustaining treatments at the end of life, including both medical technologies (e.g., the mechanical ventilator or dialysis) and medical procedures (e.g., burdensome surgeries or even CPR). Many patients fill out advance directives that include both a designated HcPOA and a LW.

However, what happens when the HcPOA's decision conflicts with the patient's LW regarding end-of-life treatment? That is - what happens when the designated person serving as the HcPOA refuses to follow the patient's treatment preferences documented in the LW? States and territories treat this

Received December 24, 2019

Accepted January 23, 2020

Published online February 4, 2020 issue differently. Ohio law (see Ohio Revised Code $\$ 2133.03(\mathrm{~B})$ ) mandates that the LW takes precedence over the HcPOA when in conflict, which is even explicitly stated in the standard Ohio advance directive form. Other states, like Maryland and North Carolina, grant patients the power to dictate whether their treatment preferences documented in the LW are to be followed strictly or if they merely serve as guidance that can be overridden by a HcPOA. The laws of many states, like Arizona, Connecticut, and Minnesota, are silent on this matter. In our state of Georgia, however, state law (see O.C.G.A. \$31-32-14(d)) dictates that when a HcPOA and a LW exist simultaneously and conflict with each other, the HcPOA takes precedence over the LW, specifically regarding any directives about withholding or withdrawing lifesustaining treatments. And while per O.C.G.A. §31-32-7(b) HcPOAs do have the duty to make decisions that are "consistent with the intentions and desires of the [patient]," to enforce this duty on a wayward HcPOA requires an interested party to petition the local courts to remove the HcPOA's decisionmaking authority, a time-intensive and inefficient process.

Georgia's treatment of this issue thus seems counterintuitive. Why defer to the HcPOA when there are documented treatment preferences from the patient herself in the LW? One justification given for this decision is that the LW is a static rather than dynamic document, and the HcPOA may have a better sense of what the patient would want at that point in time and under those specific circumstances than the previous preferences documented in the LW. The thought is that patients have difficulty accurately predicting their preferences for an uncertain future when confronted with unidentifiable conditions and unfamiliar treatments. Indeed, a 2014 systematic review of the literature regarding the stability of patients' preferences regarding life-sustaining treatments found that while the majority of patients' preferences for end-of-life care are stable over time, at least some patients do seem to experience a degree of preference changes over time. ${ }^{2}$ Further, studies have found that patients' preferences are particularly context-dependent and prone to change due to illness or hospitalization. ${ }^{3}$ Therefore, a document written in the past may not best reflect what the patient now values or what would serve her best interests.

However, the evidence does not bear out that HcPOAs are superior decision-makers compared to preferences documented in the LW. Surrogates may genuinely be unaware of the patient's preferences regarding specific medical treatments or may conflate their own preferences with the patient's 
preferences. In fact, certain studies have found that surrogate decision-makers often are influenced by their own biases and project their own values/desires onto what they believe the patient would want. ${ }^{4}$ A 2006 systematic review of the literature even found that surrogate decision-makers only accurately predict patients' preferences around $68 \%$ of the time - a decent proportion but hardly the accuracy desired for life-ordeath decisions. ${ }^{5}$ Further, other studies have found preliminary evidence that patients' preferences documented in LWs better reflect their wishes and preferences than the predictions made by their designated surrogates. ${ }^{6}$ This is additionally supported by research in recent years that has found that the majority of patients (especially those with advance directives) do have stable end-of-life preferences over time. ${ }^{2,7}$ Thus, when these findings are combined with the additional concerns regarding the accuracy of surrogate decision-making, there does not seem to be justification for prioritizing patients' HcPOAs over their LWs based upon this concern regarding the reliability of the documented preferences in LWs.

This deference to HcPOAs in Georgia and other states then ultimately risks that patients will not have their preferences and values respected due to inaccurate decision-making by the HcPOA. If patients know of this possibility, they might believe that documenting their preferences in a LW is a futile endeavor, causing them to forgo specifying treatment preferences in a LW altogether. Conversely, patients might decline to appoint a HcPOA and only fill out treatment preferences in the LW to avoid this potential conflict, which is problematic because the LW usually only becomes operative during certain end-of-life conditions (e.g., when one has a terminal condition and/or is in a state of permanent unconsciousness) and does not cover all instances where a patient is unable to make decisions for herself.

Given this evidence and the potential unintended consequences resulting from this dynamic, this legal deference to the HcPOA in Georgia and other states should be amended to either prioritize patients' LWs or grant patients the authority in advance directive documents to dictate whether their HcPOA or LW has ultimate authority regarding medical decision-making, especially at the end of life. Additionally, states whose laws are silent on this matter should remedy this situation to avoid any potential future issues arising from this loophole. Yet change is never easy and is rarely quick, which is doubly true when it comes to healthcare law and policy. Until these legislative fixes happen, residents of Georgia and other states that do not prioritize patients' LWs should take special care to ensure that they select their designated surrogate decisionmaker with the utmost consideration - preferably appointing someone who is not only aware and understanding of one's wishes regarding healthcare at the end of life, but also has the will and integrity to honor those wishes, regardless of their personal preferences.

Corresponding Author: Jordan Potter, PhD; Ethics Program Wellstar Health System, 2000 South Park Place, Atlanta, GA 30339, USA (e-mail:jordan.potter@wellstar.org).

\section{Compliance with Ethical Standards:}

Conflict of Interest: The authors declare that they do not have a conflict of interest.

\section{REFERENCES}

1. Yadav KN, Gabler NB, Cooney E, et al. Approximately one in three US adults completes any type of advance directive for end-of-life care. Health Aff. 2017; 36(7): 1244-1251.

2. Auriemma CL, Nguyen CA, Bronheim R, et al. Stability of end-of-life preferences: a systematic review of the evidence. JAMA Int Med. 2014; 174(7): 1085-1092.

3. Ditto PH, Jacobson JA, Smucker WD, Danks JH, Fagerlin A. Context changes choices: a prospective study of the effects of hospitalization on lifesustaining treatment preferences. Med. Decis. Making. 2006; 26(4): 313322 .

4. Fagerlin A, Ditto PH, Danks JH, Houts RM, Smucker WD. Projection in surrogate decisions about life-sustaining medical treatments. Health Psychol. 2001; 20(3): 166-175.

5. Shalowitz DI, Garrett-Mayer E, Wendler D. The accuracy of surrogate decision makers: a systematic review. Arch Intern Med. 2006; 166(5): 493497.

6. Bravo G, Sene M, Arcand M. Making medical decisions for an incompetent older adult when both a proxy and an advance directive are available: which is more likely to reflect the older adult's preferences? J Med Ethics. 2018; 44(7): 498-503.

7. van Wijmen MPS, Pasman HRW, Twisk JWR, Widdershoven GAM, Onwuteaka-Philipsen BD. Stability of end-of-life preferences in relation to health status and life-events: a cohort study with a 6-year follow-up among holders of an advance directive. PloS One. 2018; 13(12): e0209315.

Publisher's Note Springer Nature remains neutral with regard to jurisdictional claims in published maps and institutional affiliations. 